\title{
Estimating volatility and model parameters of stochastic volatility models with jumps using particle filter ${ }^{\star}$
}

\author{
Shin Ichi Aihara* Arunabha Bagchi ${ }^{* *}$ Saikat Saha ${ }^{* * *}$ \\ * Department of Mechanics and Systems Design, \\ Tokyo University of Science, Suwa, Toyohira Chino,Nagano, Japan, \\ (e-mail: aihara@rs.suwa.tus.ac.jp) \\ ** FELab and Department of Applied Mathematics, \\ University of Twente, The Netherlands, \\ (e-mail: a.bagchi@ewi.utwente.nl) \\ *** Department of Applied Mathematics, University of Twente, \\ The Netherlands, (e-mail: s.saha@ewi.utwente.nl)
}

\begin{abstract}
Despite the success of particle filter, there are two factors which cause difficulties in its implementation. The first one is the choice of importance functions commonly used in the literature which are far from being optimal. The second one is the combined state and parameter estimation problem. In a widely used Heston model on stochastic volatility in financial literature, we are able to circumvent both these problems. To reflect the most realistic situation, we also include jump in the stochastic volatility model. Numerical results show the effectiveness of the algorithms.
\end{abstract}

\section{INTRODUCTION}

Before starting the general framework of this paper, we start with the continuous time Heston Volatility model :

$$
\begin{aligned}
d S_{t} & =\mu_{S} S_{t} d t+\sqrt{v_{t}} S_{t} d B_{t} \\
d v_{t} & =\kappa\left(\theta-v_{t}\right) d t+\xi \sqrt{v_{t}} d Z_{t}
\end{aligned}
$$

where $v_{t}$ is the (unobserved) volatility and $S_{t}$ is the perfectly observable stock price. $B_{t}$ and $Z_{t}$ are standard Brownian motion processes with correlation $\rho$. Here, we can construct the observation process $y_{t}=\log S_{t} / S_{0}$ and corresponding stochastic differential equation for $y_{t}$ is given as

$$
d y_{t}=\left(\mu_{S}-\frac{1}{2} v_{t}\right) d t+\sqrt{v_{t}} d B_{t} .
$$

Our aim is to estimate recursively the volatility process $v_{t}$ from $\left\{y_{s}\right\}_{0 \leq s \leq t}$.

Setting

$$
\tilde{Z}_{t}=\frac{1}{\sqrt{1-\rho^{2}}}\left(Z_{t}-\rho B_{t}\right)
$$

we find that $\tilde{Z}_{t}$ is independent of $B_{t}$. Noting that

$$
\begin{aligned}
d Z_{t} & =\sqrt{1-\rho^{2}} d \tilde{Z}_{t}+\rho d B_{t} \\
& =\sqrt{1-\rho^{2}} d \tilde{Z}_{t}+\frac{\rho}{\sqrt{v_{t}}}\left(d y_{t}-\left(\mu_{S}-\frac{1}{2} v_{t}\right) d t\right),
\end{aligned}
$$

we have

\footnotetext{
$\star$ This work is partially supported by The Ministry of Education, Culture, Sports, Science and Technology of Japan under Grant-inAid for Research(C) 17560402
}

$$
\begin{gathered}
d v_{t}=\kappa\left(\theta-v_{t}\right) d t+\xi \sqrt{v_{t}} \sqrt{1-\rho^{2}} d \tilde{Z}_{t} \\
+\xi \rho\left(d y_{t}-\left(\mu_{S}-\frac{1}{2} v_{t}\right) d t\right) .
\end{gathered}
$$

The filtering problem for $v_{t}$ under $y_{t}$ is out of the usual filtering theory. See Bensoussan (1992) and Liptser (1974). The recent results for the filtering of the stochastic volatility in the continuous time framework can be found in Aihara (2006). The nonlinear filtering approach for this model ia a bit unconventional, as the state appears in the observation noise. As stated in Aihara (2006), this problem can be theoretically addressed by using Zakai equation with the splitting out method. However, the results obtained are very sensitive to the noise correlation parameter. The numerical behavior is very complicated and does not always work well.

To circumvent the above difficulty, we envisage here the use of particle filter for volatility estimation. Particle filter is a simulation based tool for filtering in discrete time framework, which can easily adapt to the nonlinearity in the model and/or non Gaussian noises. Here, the probability distributions are represented by a cloud of (weighted) particles. These particles are recursively generated from a so called "importance function", $\pi(\cdot)$. Although the resulting densities (represented by the particle clouds) do asymptotically converge to the true filtered densities as the number of particles tends to infinity, however, for finite sample size, the efficiency of this method depends heavily on the importance function used.Usually the 'naive' proposal, $p\left(v_{k} \mid v_{k-1}\right)$, which is the discrete state transition density, is used as the importance function due to the ease of drawing samples from it and corresponding simplicity of weight update (see Doucet et al. (2000)). This is recently used by Javaheri (2005) for estimating volatility, although 
in an imprecise filtering framework. However, a better choice for importance function is, $\pi=p\left(v_{k} \mid v_{k-1}^{i}, y_{k}\right)$, i.e. to make use of recent observation as it carries information about the state $v_{k}$. Moreover, as shown by Doucet et al. (2000), this is also optimal in the sense that the variance of the importance weights is minimum.

In this paper, we first consider the continuous time Heston model and apply Euler discretization scheme to this model. Next, we implement particle filter with the optimal importance function as proposed above. This procedure is then extended to the Bates model.

We also address the parameter estimation problem by augmenting the state and parameters. It is well known that this augmentation causes particle filter to work poorly. The reason is the rapid depletion of the weights associated with most of the particles (Doucet et al. (2000)). We propose here a new algorithm where the parameter is estimated by weighted average of some set of particles selected initially from any arbitrary (possibly uniform) distribution. The weights chosen come from the weight updates for the state "particles". We obtain the feasible parameter estimates without adding extra noise. Some numerical simulations are demonstrated to check the feasibility of the method proposed above.

\section{PARTICLE FILTER AND OPTIMAL IMPORTANCE FUNCTION}

\subsection{The Diffusion Model (Heston model)}

Here we present the particle filter formulation and the selection of the optimal importance function.

In order to apply the particle filter to our system, we discretize the system (4) and (3) using Euler scheme. We select this scheme mainly due to its relative simplicity and less computational load. We discretized the system as follows:

$$
\begin{aligned}
v_{k}= & v_{k-1}+\kappa\left(\theta-v_{k-1}\right) \Delta t-\xi \rho\left(\mu_{S}-\frac{1}{2} v_{k-1}\right) \Delta t \\
& +\xi \sqrt{v_{k-1}} \sqrt{1-\rho^{2}} \Delta \tilde{Z}_{k}+\xi \rho\left(y_{k}-y_{k-1}\right)
\end{aligned}
$$

and

$$
y_{k}=y_{k-1}+\left(\mu_{S}-\frac{1}{2} v_{k}\right) \Delta t+\sqrt{v_{k-1}} \Delta B_{k}
$$

where for $t_{k}-t_{k-1}=\Delta t$,

$$
\Delta B_{k}=B_{t_{k}}-B_{t_{k-1}}, \Delta \tilde{Z}_{k}=\tilde{Z}_{t_{k}}-\tilde{Z}_{t_{k-1}}
$$

Now we use the sequential importance sampling (with resampling) algorithm for the particle filter.

- The updated weight $w_{k}^{(i)}$ at $t_{k}$ is obtained by

$$
w_{k}^{(i)}=w_{k-1}^{(i)} \frac{p\left(y_{k} \mid v_{0: k}^{(i)}, y_{0: k-1}\right) p\left(v_{k}^{(i)} \mid v_{0: k-1}^{(i)}, y_{0: k-1}\right)}{\pi\left(v_{k}^{(i)} \mid v_{k-1}^{(i)}, y_{k}\right)}
$$

- It is possible to select the importance function $\pi$ as the optimal selection as in Doucet et al. (2000)

$$
\pi\left(v_{k} \mid v_{k-1}, y_{k}\right)=p\left(v_{k} \mid v_{k-1}, y_{k}\right) \text {. }
$$

- Form (5) we can easily get

$$
p\left(v_{k} \mid v_{k-1}, y_{k}\right)=\mathcal{N}\left(m\left(v_{k-1}, y_{k}\right), \sigma^{2}\left(v_{k-1}\right)\right)
$$

where

$$
\begin{aligned}
m\left(v_{k-1}, y_{k}\right)= & v_{k-1}+\kappa\left(\theta-v_{k-1}\right) \Delta t \\
& -\xi \rho\left(\mu_{S}-\frac{1}{2} v_{k-1}\right) \Delta t+\xi \rho\left(y_{k}-y_{k-1}\right)
\end{aligned}
$$

and

$$
\sigma\left(v_{k-1}\right)=\xi \sqrt{v_{k-1}} \sqrt{1-\rho^{2}} \sqrt{\Delta t}
$$

Hence we obtain the optimal importance function for the particle filter. Additionally, we enforce the hard constraint that the samples selected from this proposal are all positive.

- Next problem is to obtain $p\left(v_{k} \mid v_{0: k-1}, y_{0: k-1}\right)$. Now substituting the observation data $y_{k}$ into (5), we obtain

$$
\begin{aligned}
v_{k}= & v_{k-1}+\kappa\left(\theta-v_{k-1}\right) \Delta t-\xi \rho\left(\mu_{S}-\frac{1}{2} v_{k-1}\right) \Delta t \\
& +\xi \sqrt{v_{k-1}} \sqrt{1-\rho^{2}} \Delta \tilde{Z}_{k} \\
& +\xi \rho\left\{\left(\mu_{S}-\frac{1}{2} v_{k}\right) \Delta t+\sqrt{v_{k-1}} \Delta B_{k}\right\}
\end{aligned}
$$

i.e.,

$$
\begin{aligned}
v_{k}= & \left(1+\frac{1}{2} \xi \rho \Delta t\right)^{-1}\left\{v_{k-1}+\kappa\left(\theta-v_{k-1}\right) \Delta t\right. \\
& +\xi \rho \frac{1}{2} v_{k-1} \Delta t+\xi \sqrt{v_{k-1}} \sqrt{1-\rho^{2}} \Delta \tilde{Z}_{k} \\
& \left.+\xi \rho \sqrt{v_{k-1}} \Delta B_{k}\right\} .
\end{aligned}
$$

This implies that

$$
\begin{array}{r}
p\left(v_{k} \mid v_{0: k-1}, y_{0: k-1}\right)=p\left(v_{k} \mid v_{k-1}\right) \\
=\mathcal{N}\left(\tilde{m}\left(v_{k-1}\right), \tilde{\sigma}^{2}\left(v_{k-1}\right)\right)
\end{array}
$$

where

$$
\begin{aligned}
\tilde{m}\left(v_{k-1}\right)= & \left(1+\frac{1}{2} \xi \rho \Delta t\right)^{-1}\left\{v_{k-1}+\kappa\left(\theta-v_{k-1}\right) \Delta t\right. \\
& +\frac{\xi \rho}{2} v_{k-1} \Delta t
\end{aligned}
$$

and

$$
\tilde{\sigma}\left(v_{k-1}\right)=\left(1+\frac{1}{2} \xi \rho \Delta t\right)^{-1} \xi \sqrt{v_{k-1}} \sqrt{\Delta t} .
$$

- The likelihood function becomes

$$
p\left(y_{k} \mid v_{0: k}, y_{0: k-1}\right)=p\left(y_{k} \mid v_{k}, v_{k-1}, y_{k-1}\right)
$$

which is given as

$$
\begin{array}{r}
p\left(y_{k} \mid v_{k}, v_{k-1}, y_{k-1}\right)= \\
\mathcal{N}\left(y_{k-1}+\left(\mu_{s}-\frac{1}{2} v_{k}\right) \Delta t, v_{k-1} \Delta t\right) .
\end{array}
$$

\subsection{The Diffusion Model with Jump (Bates model)}

We adopt the Bates model ( Bates (1996)) to characterize the stock dynamics:

$$
\begin{aligned}
d S_{t} & =\mu_{S} S_{t} d t+\sqrt{v_{t}} S_{t} d B_{t}+S_{t} d Z_{t}^{J}-\lambda m^{J} S_{t} d t, \\
d v_{t} & =\kappa\left(\theta-v_{t}\right) d t+\xi \sqrt{v_{t}} d Z_{t}
\end{aligned}
$$

where $Z_{t}^{J}$ denotes the pure-jump process which contains two components: random jump-event times and random 
jump sizes. The jump-event times $\left\{T_{i} ; i \geq 1\right\}$ arrive with a constant intensity $\lambda$. Given the arrival of the i-th jump event, the stock price jumps from $S_{T_{i}^{-}}$to $S_{T_{i}^{-}} \exp \left(U^{s_{i}}\right)$ where $U_{i}^{s}$ is normally distributed with mean $\mu_{J}$ and variance $\sigma_{J}^{2}$, independent of $B_{t}$ and $Z_{t}$, inter -jump times and of $U_{j}^{s}$, for $j \neq i$. Intuitively, the conditional probability at time $t$ of another jump before $t+\Delta t$ is, for some small $\Delta t$, approximately $\lambda \Delta t$ and, conditional on a jump event, the mean relative jump size is $m^{J}=E\left(\exp \left(U^{s}\right)-1\right)=$ $\exp \left(\mu_{J}+\sigma_{J}^{2} / 2\right)-1$. Combining the effects of random jump timing and size, the last term $\lambda m^{J} S_{t} d t$ in (6) compensates for the instantaneous change in expected stock introduced by the pure-jump process $Z_{t}^{J}$.

Now by using the same approach as in the previous section, we transform (6) to $y_{t}=\log S_{t}$. By using Ito's formula, we have

$$
d y_{t}=\left(\mu_{S}-\lambda m^{J}-\frac{1}{2} v_{t}\right) d t+\sqrt{v_{t}} d B_{t}+d q_{t}^{J}
$$

where $q_{t}^{J}$ is a compound Poisson process with intensity $\lambda$ and Gaussian distribution of jump size, $\mathcal{N}\left(\mu_{J}, \sigma_{J}^{2}\right)$. Noting that

$$
\begin{aligned}
d Z_{t}= & \sqrt{1-\rho^{2}} d \tilde{Z}_{t}+\frac{\rho}{\sqrt{v_{t}}}\left(d y_{t}\right. \\
& \left.-\left(\mu_{S}-\lambda m^{J}-\frac{1}{2} v_{t}\right) d t-d q_{t}^{J}\right),
\end{aligned}
$$

we have

$$
\begin{aligned}
d v_{t}= & \kappa\left(\theta-v_{t}\right) d t+\xi \sqrt{v_{t}} \sqrt{1-\rho^{2}} d \tilde{Z}_{t} \\
& +\rho \xi\left(d y_{t}-\left(\mu_{S}-\lambda m^{J}-\frac{1}{2} v_{t}\right) d t-d q_{t}^{J}\right) .
\end{aligned}
$$

Now discretize these systems as

$$
\begin{aligned}
y_{k}-y_{k-1}= & \left(\mu_{S}-\lambda m^{J}-\frac{1}{2} v_{k}\right) \Delta t \\
& +\sqrt{v_{k-1}} \Delta B_{k}+\Delta q_{k}^{J},
\end{aligned}
$$

where $\Delta q_{k}^{J}$ is the jump in $q_{t_{k}}^{J}$. We also have

$$
\begin{aligned}
& v_{k}-v_{k-1}=\kappa\left(\theta-v_{k-1}\right) \Delta t+\xi \sqrt{v_{k-1}} \sqrt{1-\rho^{2}} \Delta \tilde{Z}_{k} \\
& +\xi \rho\left(y_{k}-y_{k-1}-\left(\mu_{S}-\lambda m^{J}-\frac{1}{2} v_{k-1}\right) \Delta t-\Delta q_{k}^{J}\right) .
\end{aligned}
$$

Now in this diffusion-jump case we need to derive the explicit form of the weight update equation:

- The updated weight $w_{k}^{(i)}$ is obtained in the same way as in the previous section:

$$
w_{k}^{(i)}=w_{k-1}^{(i)} \frac{p\left(y_{k} \mid v_{0: k}^{(i)}, y_{0: k-1}\right) p\left(v_{k}^{(i)} \mid v_{0: k-1}^{(i)}, y_{0: k-1}\right)}{\pi\left(v_{k}^{(i)} \mid v_{k-1}^{(i)}, y_{k}\right)} .
$$

- Assuming that at $t_{k}$ the jump occurs at most one, we have from Cont (2004)

$$
\begin{aligned}
p\left(v \mid v_{k-1}, y_{k}\right)= & \left\{\frac{\left(1-e^{-\lambda \Delta t} \lambda \Delta t\right)}{\sqrt{2 \pi \bar{\sigma}^{2}\left(v_{k-1}\right)}}\right. \\
& \times \exp \left[-\frac{\left(v_{k}-\bar{m}\left(v_{k-1}, y_{k}\right)\right)^{2}}{2 \bar{\sigma}^{2}\left(v_{k-1}\right)}\right] \\
& +\frac{e^{-\lambda \Delta t} \lambda \Delta t}{\sqrt{2 \pi\left(\bar{\sigma}^{2}\left(v_{k-1}\right)+\xi^{2} \rho^{2} \sigma_{J}^{2}\right)}}
\end{aligned}
$$

$$
\left.\times \exp \left[-\frac{\left(v_{k}-\bar{m}\left(v_{k-1}, y_{k}\right)+\xi \rho \mu_{J}\right)^{2}}{2\left(\bar{\sigma}^{2}\left(v_{k-1}\right)+\xi^{2} \rho^{2} \sigma_{J}^{2}\right)}\right]\right\}
$$

where

$$
\begin{aligned}
& \bar{m}\left(v_{k-1}, y_{k}\right)=v_{k-1}+\kappa\left(\theta-v_{k-1}\right) \Delta t \\
& +\rho \xi\left(y_{k}-y_{k-1}-\left(\mu_{S}-\lambda m^{J}-\frac{1}{2} v_{k-1}\right) \Delta t\right)
\end{aligned}
$$

and

$$
\bar{\sigma}^{2}\left(v_{k-1}\right)=\xi^{2} v_{k-1}\left(1-\rho^{2}\right) \Delta t .
$$

- Now we shall evaluate $p\left(v_{k} \mid v_{0: k-1}, y_{0: k-1}\right)$. Substituting $y_{k}-y_{k-1}$ into (10), we get

$$
\begin{aligned}
v_{k}= & \left(1+\frac{1}{2} \xi \rho \Delta t\right)^{-1}\left\{v_{k-1}+\kappa\left(\theta-v_{k-1}\right) \Delta t\right. \\
& -\xi \rho\left(\mu_{S}-\frac{1}{2} v_{k-1}\right) \Delta t+\xi \sqrt{v_{k-1}} \sqrt{1-\rho^{2}} \Delta \tilde{Z}_{k} \\
& \left.+\xi \rho\left\{\mu_{S} \Delta t+\sqrt{v_{k-1}} \Delta B_{k}\right\}\right\} .
\end{aligned}
$$

Hence, $p\left(v_{k} \mid v_{0: k-1}, y_{0: k-1}\right)=p\left(v_{k} \mid v_{k-1}\right)$, which is same as the non-jump case. This is a quite reasonable result because $v_{k}$ does not contain any jumps.

$$
p\left(v_{k} \mid v_{k-1}\right)=\mathcal{N}\left(\tilde{m}\left(v_{k-1}\right), \tilde{\sigma}\left(v_{k-1}\right)\right)
$$

where

$$
\begin{aligned}
\tilde{m}\left(v_{k-1}\right)= & \left(1+\frac{1}{2} \xi \rho \Delta t\right)^{-1}\left\{v_{k-1}+\left(\omega-\theta v_{k-1}\right) \Delta t\right. \\
& \left.-\xi \rho\left(\mu_{S}-\frac{1}{2} v_{k-1}\right) \Delta t+\xi \rho \mu_{S} \Delta t\right\}
\end{aligned}
$$

and

$$
\tilde{\sigma}\left(v_{k-1}\right)=\left(1+\frac{1}{2} \xi \rho \Delta t\right)^{-1} \xi \sqrt{v_{k-1}} \sqrt{\Delta t} .
$$

- We need to derive the explicit form of $p\left(y_{k} \mid v_{0: k}\right.$, $\left.y_{0: k-1}\right)$. It is easy to show that

$$
p\left(y_{k} \mid v_{0: k}, y_{0: k-1}\right)=p\left(y_{k} \mid y_{k-1}, v_{k}, v_{k-1}\right) \text {. }
$$

One notes

$$
\begin{gathered}
p\left(y_{k} \mid y_{k-1}, v_{k-1: k}\right)=\frac{\left(1-e^{-\lambda \Delta t} \lambda \Delta t\right)}{\sqrt{2 \pi v_{k-1} \Delta t}} \\
\times \exp \left(-\frac{\left(y_{k}-y_{k-1}-\left(\mu_{S}-\lambda m^{J}-\frac{1}{2} v_{k}\right) \Delta t-u_{k}\right)^{2}}{2 v_{k-1} \Delta t}\right) \\
+\frac{e^{-\lambda \Delta t} \lambda \Delta t}{\sqrt{2 \pi\left(v_{k-1} \Delta t+\sigma_{J}^{2}\right)}} \\
\times \exp \left(-\frac{\left(y_{k}-y_{k-1}-\left(\mu_{S}-\lambda m^{J}-\frac{1}{2} v_{k}\right) \Delta t-u_{k}-\mu_{J}\right)^{2}}{2\left(v_{k-1} \Delta t+\sigma_{J}^{2}\right)}\right) .
\end{gathered}
$$

where

$$
u_{k}=\frac{\rho}{\xi}\left[v_{k}-v_{k-1}-\kappa\left(\theta-v_{k-1}\right) \Delta t\right]
$$

\section{PARAMETER IDENTIFICATION PROBLEM}

To identify the parameters contained in the system model, we construct the augmented state $z_{k}=\left(v_{k}, \alpha\right)$ where for the diffusion case, vector $\alpha$ contains the parameters as

$$
\alpha=\left[\begin{array}{llll}
\kappa & \theta & \xi & \mu_{S} \rho
\end{array}\right]
$$

and similarly, for the jump-diffusion case

$$
\alpha=\left[\kappa \theta \xi \mu_{S} \rho \lambda \mu_{J} \sigma_{J}\right] \text {. }
$$

To perform the particle filter for $z_{k}$ we assume that $\alpha \in U$ (uniform distribution with known upper and lower 
bounds), and is independent of the initial distribution of $v_{1}$ which is taken here as Gaussian. Hence we can apply the particle filter algorithm developed in the previous section to $z_{k}$-process. Noting that the state $\alpha$ is time independent, the parameter value $\alpha^{(i)}$ is not updated and we encounter the degenerated problem. In this paper to avoid this deficiency, we use the simple random resampling for each parameter and apply the systematic resampling for the state $v_{k}$. Repeating this resampling for every parameter, we observe that the estimated parameters do not degenerate. From the simulation results, we find that the random resampling for the parameters performs similar effect as adding the so called roughening noises to the parameter values.

\section{SIMULATION STUDIES}

For our numerical studies, we consider only the diffusion model with jumps which represents the most realistic scenario. In the subsequent simulations, resampling is done whenever the effective sample size as defined in Doucet et al. (2000) falls below two-third of the sample size used. To check the algorithm proposed here, the stock price and volatility process are simulated using the following values of the parameters

$$
\begin{gathered}
\kappa=3.0, \theta=0.1, \mu=0.1, \rho=-0.2, \\
\xi=0.4, \lambda=4.5, \mu_{J}=-0.1, \sigma_{J}=0.1 .
\end{gathered}
$$

The simulated volatility and the log price $y(t)$ are shown in Fig.1. and Fig.2 respectively. In this simulation studies, we set $\Delta t=0.001$ and the number of particles is set as 2000 .

For unknown parameters, we set

$$
\begin{array}{r}
\kappa \in U[1,10], \quad \theta \in U[0.05,0.5], \quad \mu \in U[0.05,0.3], \\
\xi \in U[0.01,0.91], \quad \rho \in U[-0.8,0] \\
\lambda \in U[0,5], \quad \mu_{J} \in U[-0.2,0], \quad \sigma_{J} \in U[0,0.2] .
\end{array}
$$

We also set

$$
v_{1} \in \mathcal{N}\left(0.25,0.02^{2}\right)
$$

The true and estimated volatility are demonstrated in Fig.3 and its square error is also shown in Fig.4.

The estimates of unknown parameters are also demonstrated in Figs.5 to 12. In these simulation studies, one may find bias in some of the estimated parameters, which occurs depending on the setting of the upper and lower bounds selected for the estimation of unknown parameters.

\section{CONCLUSION}

For the discretized Heston model with jumps, we derive the stochastic volatility using particle filter algorithm with the optimal importance function. By using the simple random resampling method, we also propose an estimation procedure for the parameters of the model. In this setting, with these estimated parameters, the estimation of stochastic volatility works quite well. So, one can expect these estimated parameters to be quite reasonable. Comparing the estimated parameters with other known estimation

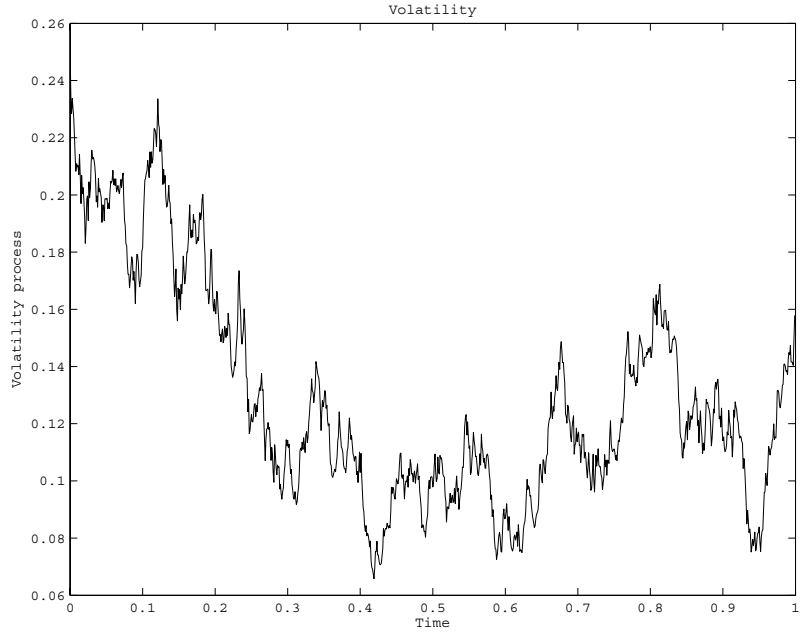

Fig. 1. Simulated volatility

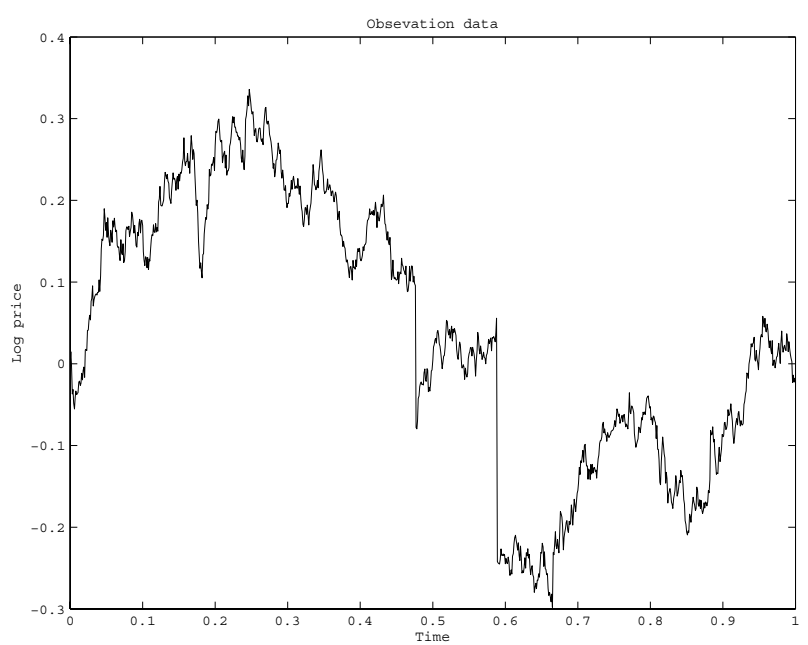

Fig. 2. Observation data (log price)

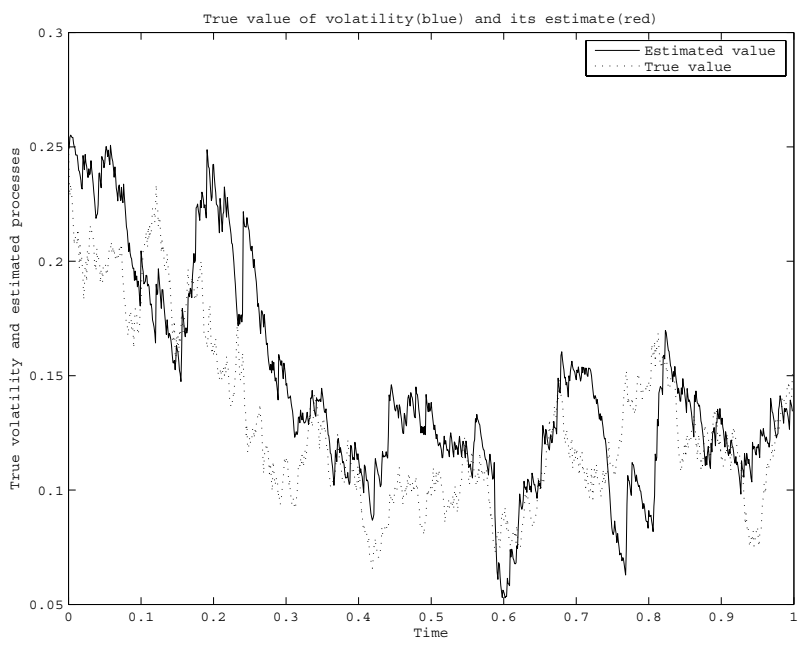

Fig. 3. True and estimated volatility processes 


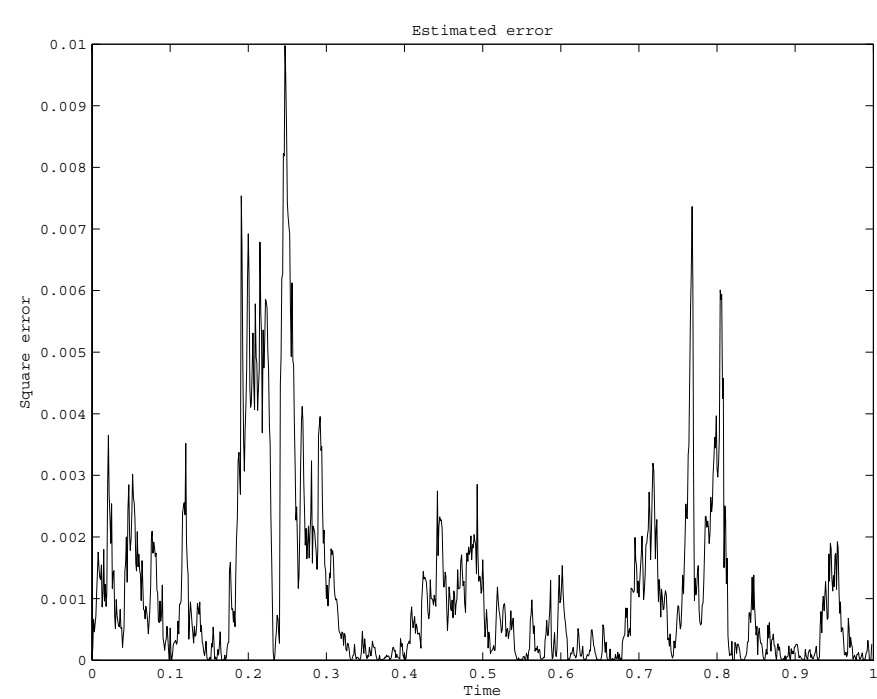

Fig. 4. Square error of estimate $v$

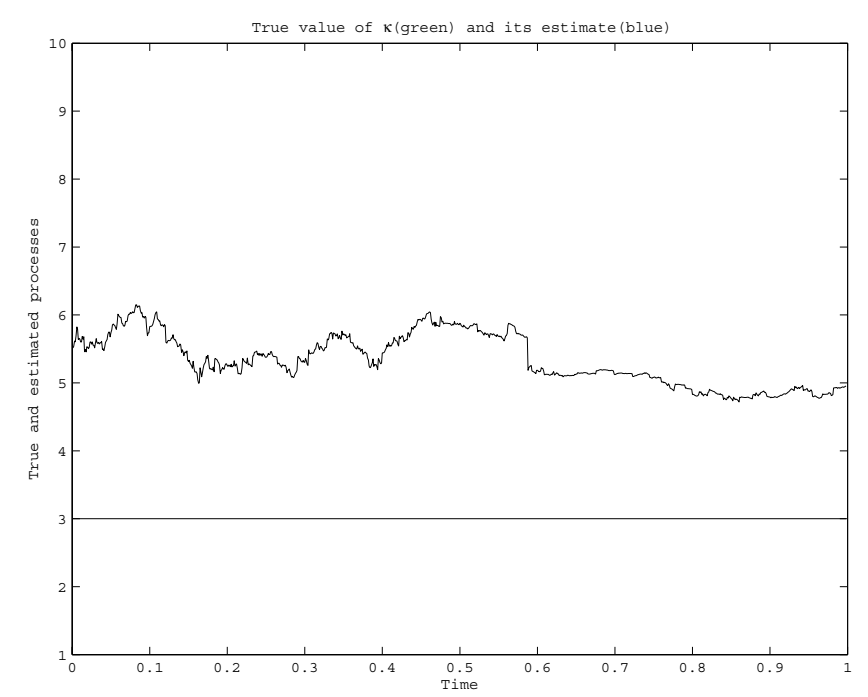

Fig. 5. True and estimated $\kappa$

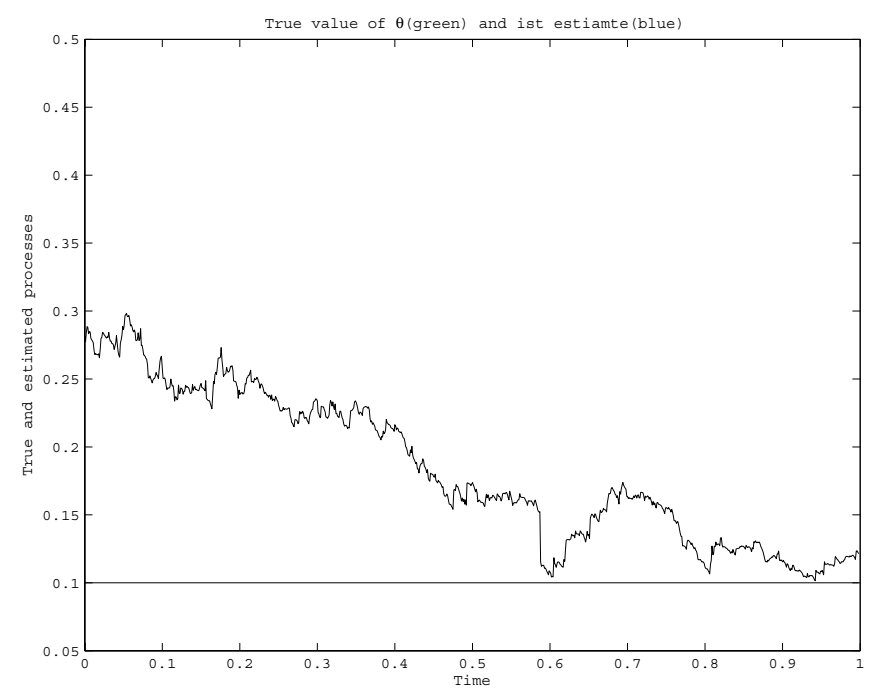

Fig. 6. True and estimated $\theta$

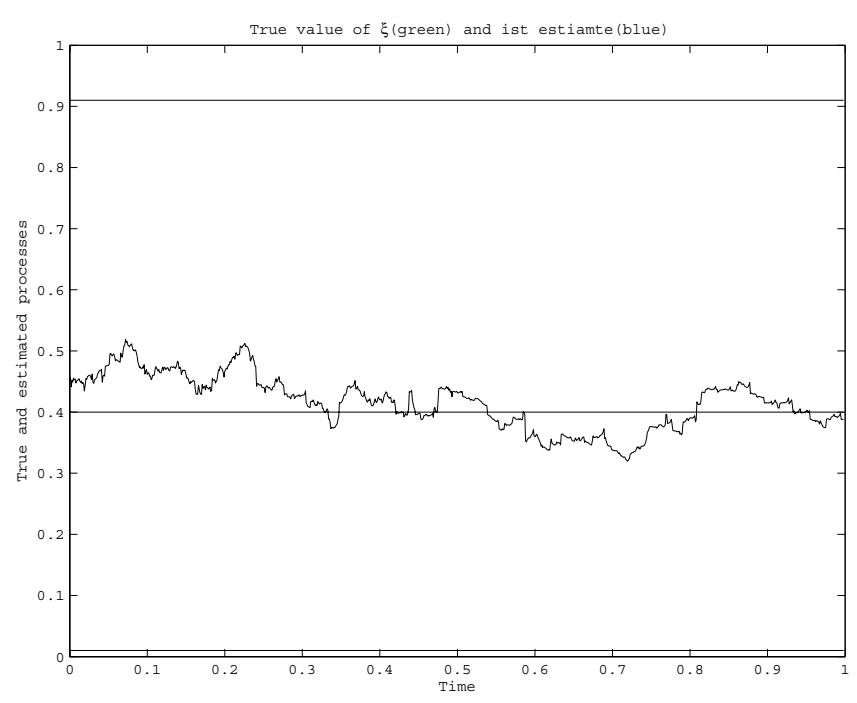

Fig. 7. True and estimated $\xi$

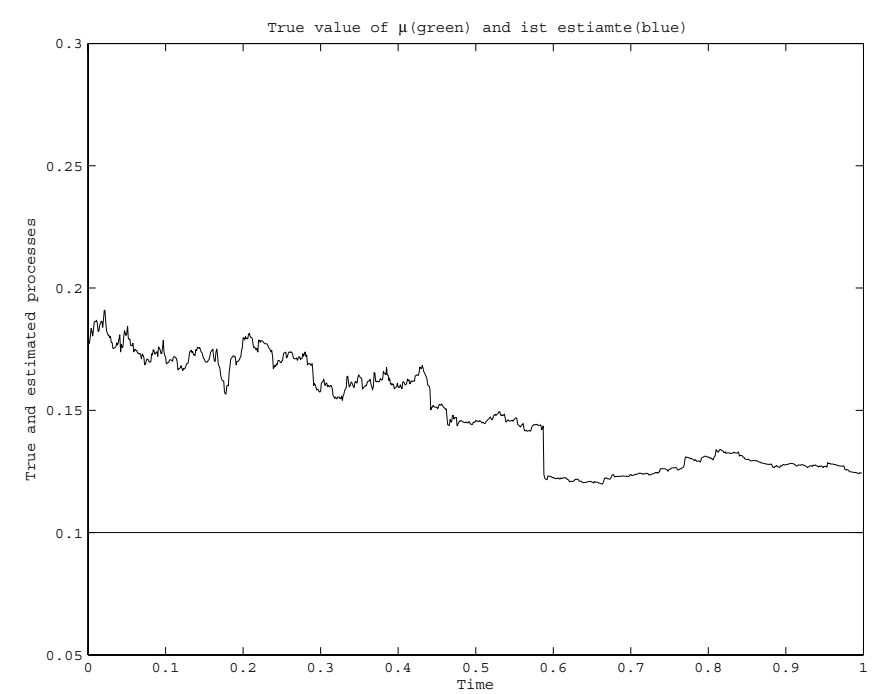

Fig. 8. True and estimated $\mu$

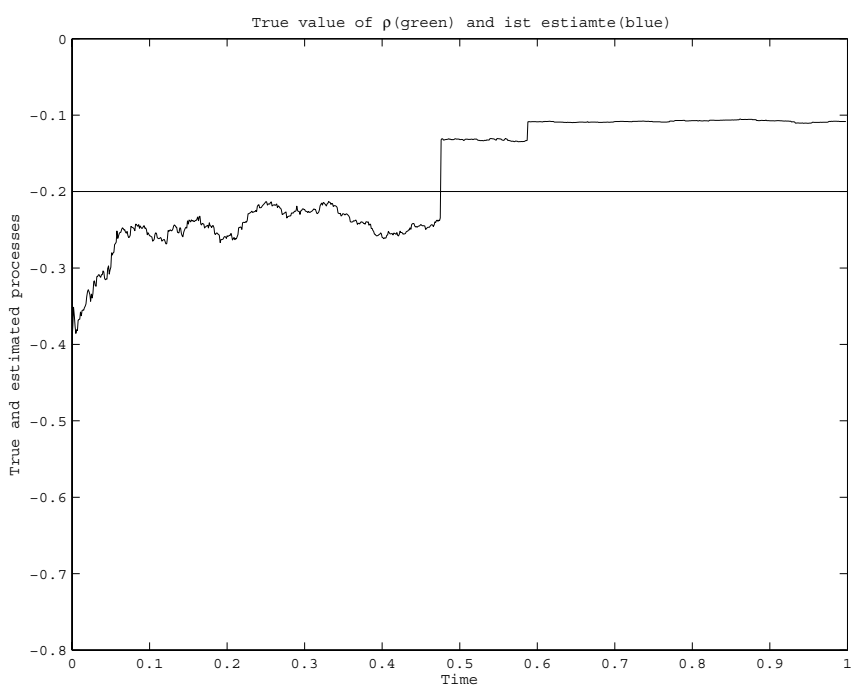

Fig. 9. True and estimated $\rho$ 


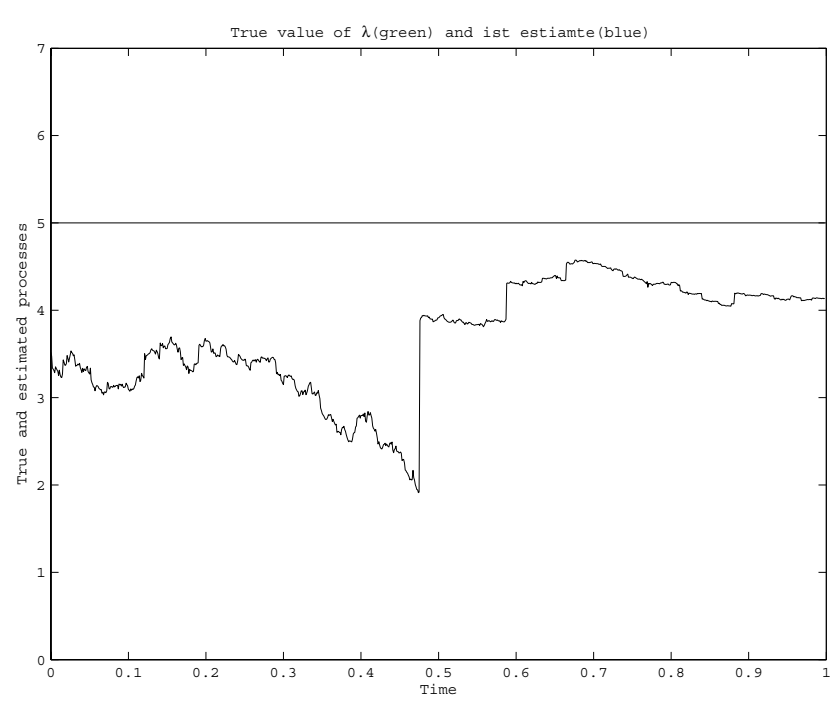

Fig. 10. True and estimated $\lambda$

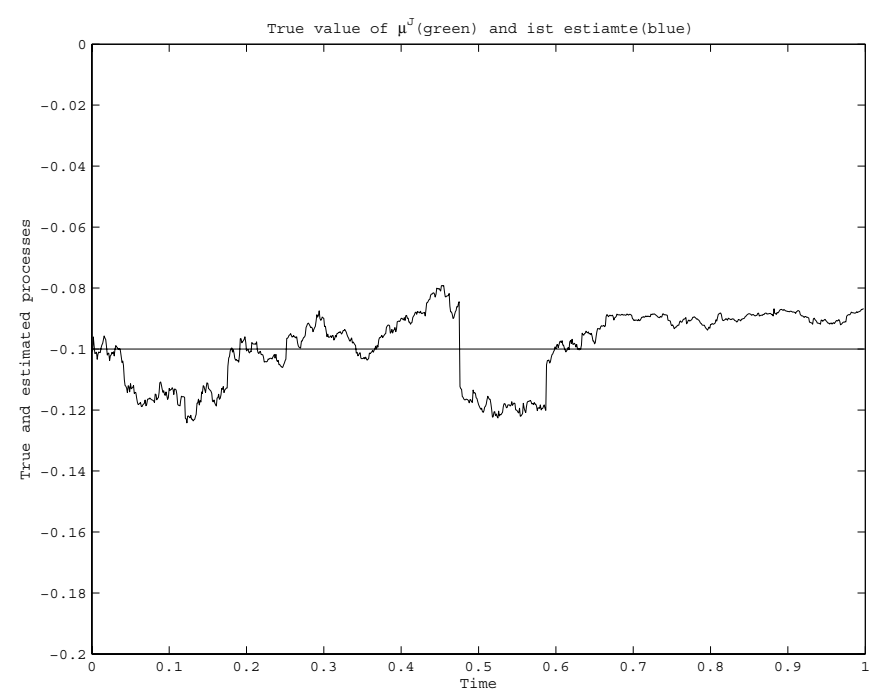

Fig. 11. True and estimated $\mu_{J}$

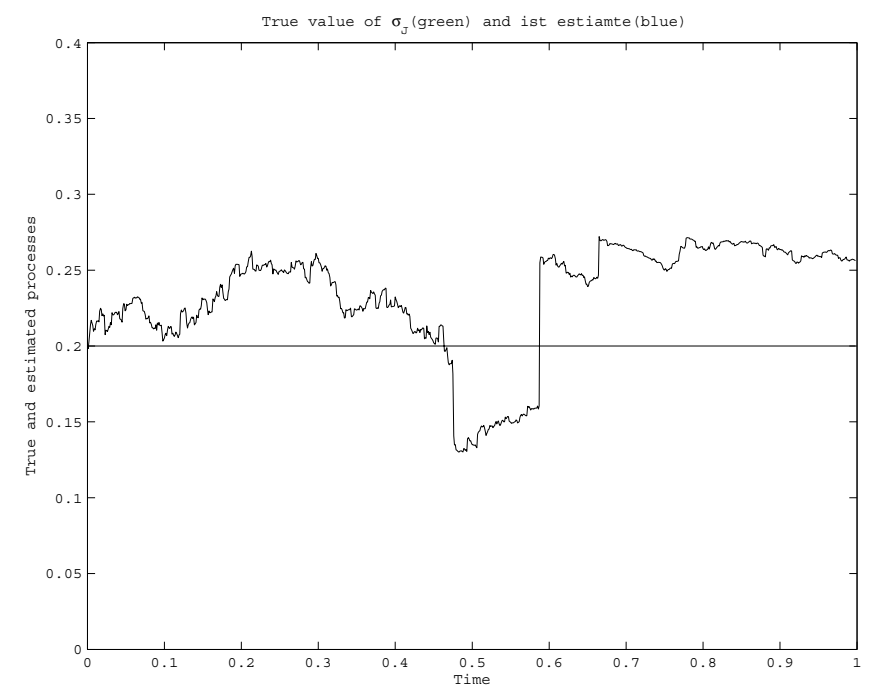

Fig. 12. True and estimated $\sigma_{J}$ techniques is out of the scope for our current work and will be discussed elsewhere in the future.

\section{REFERENCES}

A. Bensoussan. Stochastic Control of Partially Observable Systems. Cambridge University Press, Cambridge, 1992. R.S. Liptser and A.N. Shiryaev. Statistics of Random Processes. Springer-Verlag, New York, 1974.

S. Aihara and A. Bagchi. Filtering and identification of Heston's stochastic volatility and its market risk. J. Economical Dynamics and Control,30(12) 2006.

A.Javaheri. Inside Volatility Arbitrage. John Wiley \& Sons, Inc., Hoboken, 2005.

M.S. Arulampalam, S. Maskell, N. Gordon, and T. Clapp. A tutorial on particle filters for online nonlinear /nonGaussian Bayesian tracking. IEEE Trans on Signal Processing, 50, 2002.

A. Doucet, S. Godsill and C. Andrieu. On sequential Monte Carlo sampling methods for Bayesian filtering. Statistics and Computing, 10, 2000.

D. Bates. Jumps and stochastic volatility: the exchange rate processes implicit in deutschemark option. Rev. Fin. Studies, 9, 1996.

R. Cont and P. Tankov. Financial Modelling With Jump Processes. Chapman \& Hall/CRC, Boca Raton, 2004. 